\title{
Hidden Explosive Detection Systems for Vehicle
}

\author{
Yashwant Kurmi \\ Department of ECE, \\ Maulana Azad \\ National Institute of Technology \\ Bhopal, India
}

\author{
Vijayshri Chaurasia \\ Department of ECE, \\ Maulana Azad \\ National Institute of Technology \\ Bhopal, India
}

\begin{abstract}
The hidden explosive material detection is a non-destructive investigation practice to identify explosive substances. It is widely deployed at airports, harbours, and sensitive areas of country specially the border areas. The basic detection arrangements are improving at their own pace but unauthorized movement of arms and illegal imports still happens. It is very common through vehicles by making false voids in their fuel tank or inside door panels. The hidden voids detection is a difficult task inside the compartments of vehicle. It is also unreliable, time consuming, and expensive. In conventional detection systems, canines and $\mathrm{x}$-rays are the most common methods. Many advancements in the mentioned field have been proposed. This paper gives a comparative analysis of most significant explosive detection systems for identifying vehicle voids and compartments that may have explosives or illegal imports..
\end{abstract}

\section{Keywords}

Laser Vibrometer, Hide Explosive Detection, Tetra Hertz Detection system, Spectrum based IED.

\section{INTRODUCTION}

In present era, anti-social elements are using explosive materials for mass destruction. Control of such activities is very big issue for authorities. To stop the misuse of the explosive it is extremely essential to intensely inspect the corporeal belongings.

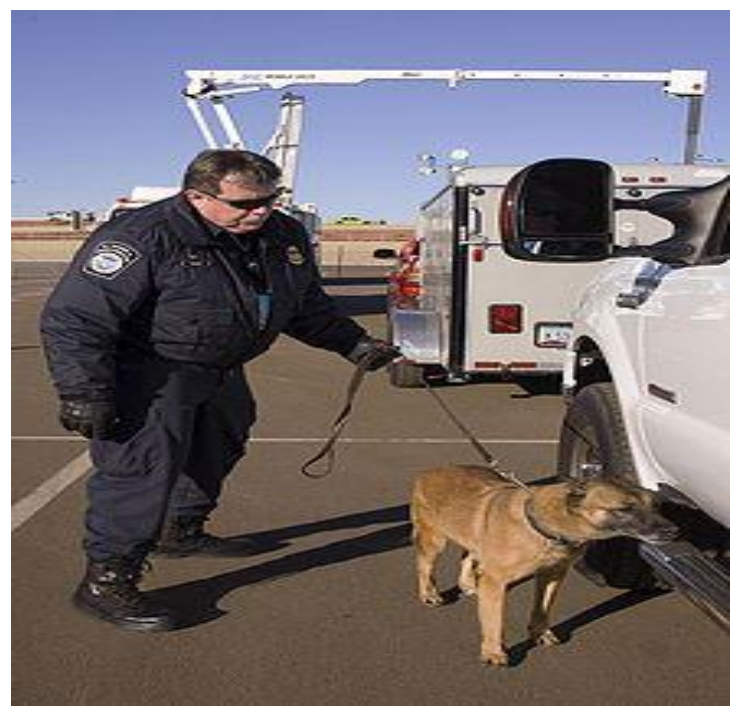

Fig.1 A U.S. Customs and Border Protection officer with an explosive-detection $\operatorname{dog}[5]$

The investigation must be done to scrutinize and define the procedural experiment in identifying the Improvised Explosive Devices (IEDs). The most movable equipments of detection have been intended for security at airport and are attentive on finding recent plastic made explosives that create the significant destruction, even in trivial quantities. The most the present explosive ingredient mainly comprises of solid, nitrogenous compounds. As enumerated in table 1. A conventional method of an explosive detection with the help of canine is as shown in fig. 1. This system is very expensive and not an automated. To make it automatic the researchers are working the different possible options. The accessibility and reliability of canine procedure is limited. The detection process through employment of $\mathrm{x}$-rays is reliable but it is expensive and not easily available. Moreover, these systems do not permit the user to augment or reduce search efforts according to threat levels for keeping the balance between thorough search capabilities and lengthy examine times.

Table 1. Common Explosive Material

\begin{tabular}{|c|c|}
\hline NAME & CHEMACAL NAME \\
\hline RDX & Cyclotrimethylenetrinitramine \\
\hline TNT & Trinitrotoluene \\
\hline PETN & Pentaerythritol Tetranitrate \\
\hline ANAL & $\begin{array}{c}\text { Ammonium Nitrate and Aluminum } \\
\text { Powder }\end{array}$ \\
\hline Dynamite & $\begin{array}{c}\text { Mixture of nitroglycerine and } \\
\text { nitrocellulose }\end{array}$ \\
\hline ANFO & Ammonium Nitrate and Fuel Oil \\
\hline Black & $\begin{array}{c}\text { Gun powder or grain powder } \\
\text { Powder }\end{array}$ \\
\hline HMX & Hexamethylene Triperoxide Diamine \\
\hline TATP & Triacetone Triperoxide \\
\hline C4 & $90 \%$ RDX+ Plasticizer \\
\hline
\end{tabular}

There exist numeral Hide Explosive recognition schemes based on vapor and trace adulteration. Krausa [13] said in his research that the materials that are volatile in nature with high vapor pressure are easily detectable [13]. But most of the current explosive composite exhibits very low pressure of vapor which makes vapor-based finding difficult. The materials of low pressure and high explosive capacity are considered the greatest threat to aviation security. However, there are a number of other materials that are used in IEDs. Cottrell has introduced the Contra band Detection System based on laser vibrometer [3]. That is more efficient for vehicle void detection with high range. Various explosive detectors technologies are used in present time. Some significant of them are considered here for this study. In rest of the paper, the section II represents Ion Mobility Spectrometry system. The part III covers the pulsed Tetra Hertz (THz) detection method, section IV give the description of Acoustic Wave's Explosive Detection, and sector V covers the Laser Vibrometer for explosive detection. Finally section VI concludes the paper.

\section{ION MOBILITY SPECTROMETRY}

The Explosive substance recognition through Ion Mobility Spectrometry (IMS) is based on the ion velocities in an 
unchanging electrically powered field. The detecting knowledge also necessitates the sample ionization of explosive materials that is basically proficient with Nickel-63 or Americium-241 radioactive materials [5].

\section{TERAHERTZ SYSTEMS}

The Tetra Hertz (THz) method is fundamentally broadband, with emanated power spread over some $\mathrm{THz}$ (usually 0.1-4 $\mathrm{THz}$ ). The $\mathrm{THz}$ Detection system by using pulsated $\mathrm{THz}$ emanation in antennas based on photoconductivity. It is formed when the density of current $\mathrm{J}$ of a subjective semiconductor is controlled in sub-picosecond times $\mathrm{ETHz}$ OC $(\mathrm{dj} / \mathrm{dt})[6]$.

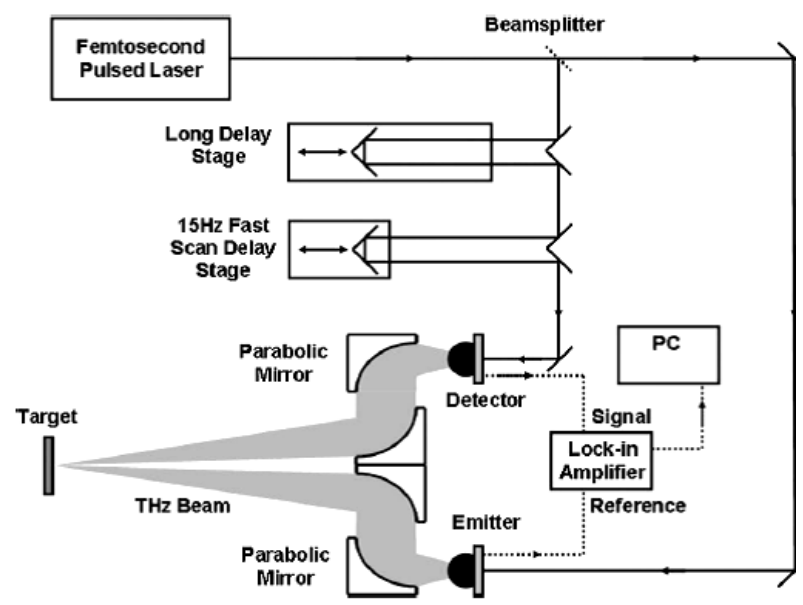

Fig 2:A THz frequency system based on photoconductivity [2].

This method consists of the various components in its complete system arrangement shown in fig. 2 . The femtoscale pulsed laser for generation of the signal. Various number of beam splitters are required for the beam deflection. The parabolic mirror combination is used for the $\mathrm{THz}$ beam to focus on the target and collection of the beam from the target for further processing. The processing unit then makes the decision on the suspected material. This system is precisely planned for the stalemate explosives material finding, this high frequency $\mathrm{THz}$ stream of light is employed for echo spectroscopy from substantial target [2].

\section{ACOUSTIC WAVE'S EXPLOSIVE DETECTION}

An arrangement for detecting the presence of an explosive comprises a pulsed intensive energy basis located at a target distance away from a substrate, the magnitude of energy is sufficient to release the internal energy of an explosive. If it is present on the substrate and thereby generate an acoustic wave [4]. On the basis of reflected acoustic wave's observation, the decision is taken on explosives. This system requires high energy that the nearby material should generate the waves for observation. The system is complex and of low efficiency.

\section{EXPLOSIVE DETECTION USING LASER VIBROMETER}

The arrangement for explosives detection (ED) and illegal imports in a vehicle includes at slightest one Laser Vibrometer (LV) to measure the vehicle vibrations.

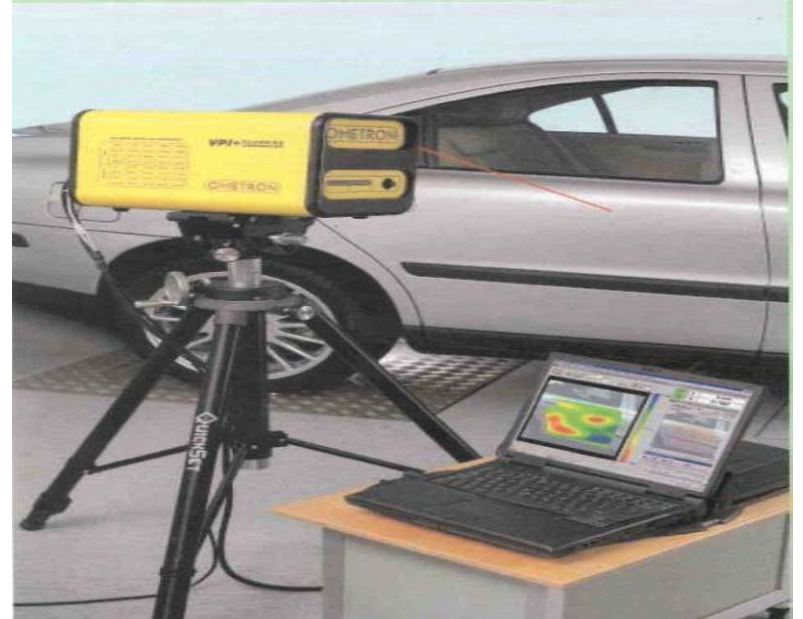

Fig.3. Simply portable system that identifies voids concealed inside vehicle lively (by taking approx. $10 \mathrm{sec}$.)

[1]

The LV measures the level of vibrations of an operating vehicle at the various points on vehicle. The LV may be directed to various points of a vehicle such as; fuel tank to measure the vibrations. Fig. 3 illustrates the basic system for the laser vibrometer. This system consists of basic signal generator system suing the oscillators. The generated signal strikes on the target in the form of a beam of signal. The reflected beam is collected by the receiving system and provided to the processor for further processing [1]

The processor compares the beam fluctuation on the basis of vehicles vibration. The database is kept in the processor for the comparative analysis of the vehicle's vibrations. On the basis of vehicle vibration the decision is made about the existence of suspected explosives inside the vehicle. One LV can be used with the other LV and sequentially directed to pre-determined points on the vehicle [3]. On a plurality LV could be used with each LV illuminating the vehicle simultaneously. The range of detection distance can be about $1 \mathrm{~cm}$ to 1000 meters [4]. Fast detection of dangerous explosive activity from a distance has many security applications [4]. One LV can be sequentially directed to various points on the vehicle corresponding to a predetermined sole point or pattern. A combination of LVs could be used to concurrently illuminate the vehicle. Next to the measurement, the vehicle vibrations are equated to a record of reference vibrations, which were taken of similar vehicles. The dignified vibrations are compared to vibrations pattern for same type of vehicle [1]. If vibration patterns of current taken exhibit differences in frequency peaks that exceed prearranged parameters, then system alerts to the operator. In this situation a more comprehensive inspection of the vehicle can be accomplished [3]. The fig. 4 illustrates the comparative analysis of the vehicles without suspected material hidden through red colour and with suspected explosive material in the inside compartment of the vehicle through blue colour as a mark for understanding. 
Measurement from vehicle with hidden explosive inside

Vibrations from vehicle with no hidden explosives inside the compartment

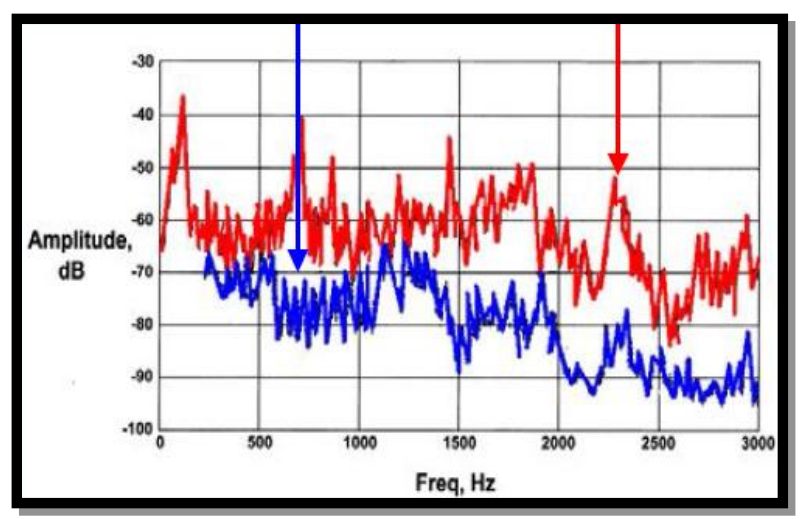

Fig 4: The LV measure vibrations and match the measurements [1]

The study of signal strength in laboratory were performed with materials dinitrotoluene (DNT), also with trinitrotoluene (TNT), and the RDX (Hexahydro-13, 5-trinitro-1,3,5-triazine, the explosive component of $\mathrm{C} 4$ plastic explosive). The other one TNT sample was a trace deposit, whereas the other explosives materials are taken in bulk amounts.

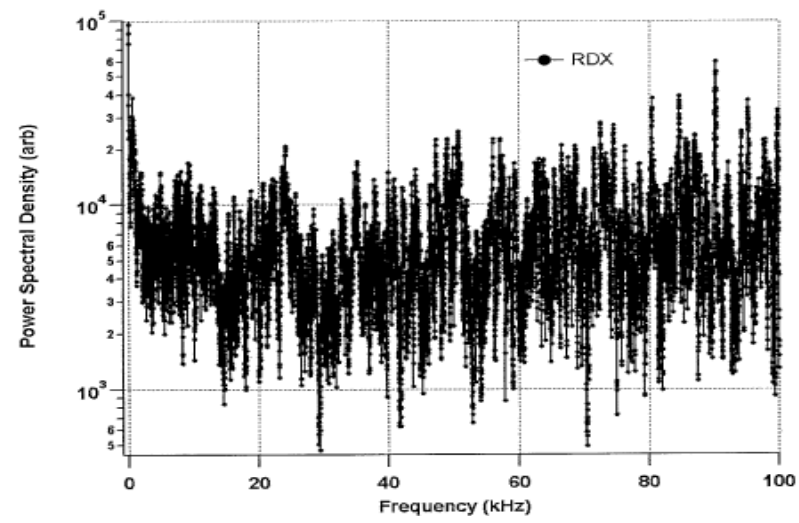

Fig 5: The response of spectrum for RDX detection [4]

The 250 can laser pulses when; approx. 7 ns duration with a fluence at target of about $15 \mathrm{~mJ} /\left[\mathrm{cm} \rrbracket^{\wedge} 2\right.$. The acoustic detector was a microphone, (Earthworks M30 HDM, Milford. N.H.) With at response that decreased beyond $30 \mathrm{kHz}$, which was positioned at a detection distance of about 4 inches from the sample [4]. A substantial signal can be observed from DNT, RDX, and TNT. The analysis of Spectrum of the signals identified by LV from the explosive RDX is revealed in Fig. 5.The Spectrum based IED is applied to substantial signal detection up to about $100 \mathrm{kHz}$, which is the resolution of the system. The marked dots are the notation for the detection of the RDX identification in the vehicle at the distance of 1 meter approximately. Approximately same results are produced by the measurements of TNT and DNT; meanwhile 1000 times weaker signals are produced for nonexplosives materials [4].

\section{CONCLUSION}

The ED System must be able to identify the suspected material cavities privileged to the vehicles deprived of applying the $\mathrm{x}$-rays. The toughness side by side is amendable, permitting the users to vary the detection severity toward toughness during great menace stages and ease in flexibility during lower risk stages. The $\mathrm{THz}$ system is very much expensive, and the acoustic wave system is complex in workability. Both the above systems are of low range as compared to the LV system. The device LV is economical, portable and easily accessible than the other recent ED recognition methods. The LV detection is non-invasive system with the capability to adjust search toughness according to risk stages. The latest laser vibrometer technique performance is proved to be the best among all the method for vehicles Explosive Detection. The image fusion analysis have a more accurate description of the scene, may also be applied for machine perception [12].

\section{REFERENCES}

[1] U.S Patent Application 12/329,726 (Explosives and contraband detection system).

[2] C. Baker, T. Lo, W. R. Tribe, B. E. Cole, M. R. Hogbin, and M. C. Kemp "Detection of concealed Explosives at a distance using Tetrahertz Technology" IEEE vol. 95, No. 8, August 2007.

[3] D. Cottrell, E. Cajon. "Explosive and Contraband Detection Syatem" US 8,482,408 B1,filingi date Dec.8, 2008, Patent date Jul.9, 2013.

[4] C. M. Wynn, R. W. Haupt, S. Kushik, and S. T. Palmacci "Method and Kit for Stand-off Detection of Explosives" 2011US 8,935,960 B2, patent filed date Jan. 20, patent date Jan. 20, 2015.

[5] https://en.wikipedia.org/wiki/Explosive_detection.

[6] P. R. Smith, D. H. Auston, and M. C. Nuss, "Subpicosecond photoconducting dipole antennas," IEEE JQE, vol. 24, no. 2, pp. 255-260, 1988.

[7] Z. Piao, M. Tani, and K. Sakai, "Carrier dynamics and terahertz radiation in photoconductive antennas," Jpn. J. AppL Phys., vol. 39, no. 1, pp. 96-100, 2000.

[8] M. Tani, K. Sakai, and H. Mimura, "Ultra-fast photoconductive detectors based on semi-insulating GaAs and InP," JJAP, vol. 36, no. 2, pp. L1175-L1178, 1997.

[9] S. Kono, M. Tani, P. Cu, and K. Sakai, "Detection of up to $20 \mathrm{THz}$ with a low-temperature-grown GaAs photoconductive antenna gated with 15 fs light pulses," Appr. Phys. Lett., vol. 77, no. 25, pp. 4104-4106, 2000.

[10] C. Baker, 1. S. Gregory, W. R. Tribe, I. V. Bradley, M. J. Evans, M. Withers, P. F. Taday, V. P. Wallace, E. H. Linfield, A. G. Davies, and M. Missous, "Terahertz puked imaging with 1.06 p.m laser excitation," Appr. Phys. Lea., vol. 83, no. 20, pp. 4113-4115, 2003.

[11] I. S. Gregory, C. Baker, W. R. Tribe, M. J. Evans, H. E. Beere, E. H. Linfield, A. G. Davies, and M. Missous, "High resistivity annealed bw temperature GaAs with 100 fs lifetimes," Appl. Phys. Lett., vol. 83, no. 20, pp. 4199-4201, 2003.

[12] Y. Kurmi, V. Chaurasia, "An Image Fusion Approach based on Adaptive Fuzzy Logic Model with Local Level Processing” IJCA (0975 - 8887) Vol. 124 - No.1, Aug. 2015.

[13] M. Krausa "Vapour and Trace Detection of Explosives for Anti-Terrorism Purposes" Springer Science \& Business Media, 06-Nov-2012. 


\section{AUTHOR PROFILE}

Yashwant Kurmi received the B.E. degree in Electronic and Communication Engineering from JEC Jabalpur, RGPV University of Bhopal, in year 2006. He acquired his Masters of Digital communication in 2013 from Maulana Azad National Institute of Technology (MANIT), Bhopal. Presently he is a Research Fellow in the department of Electronic and Communication Engineering of MANIT, Bhopal, India. He has 5 international Journals and one patent. His research interest includes image compression, image fusion, object detection and antenna design.

Vijayshri Chaurasia received the Bachelor's Degree in Electronics \& Telecommunication Engineering from the Pt.
Ravishankar Shukla University, Raipur, India, in 2002, the M.Tech. Degree in Digital Communication from Maulana Azad National Institute of Technology (MANIT), Bhopal, India in 2005. She carried out her research in image compression and awarded Ph.D. degree in 2011 from MANIT, Bhopal, India. Presently she is with MANIT, Bhopal, India. She has published more than 35 research paper of national and international repute, one patent and associated with professional bodies IETE, ISTE and IEEE. Her research interests include image processing, signal processing, pattern recognition and feature extraction 